\title{
Reproductive health in domestic animals
}

\author{
Niels Einer-Jensen and Ronald H F Hunter
}

\section{Introduction}

This review article is targeted at readers with knowledge of human reproductive health and it attempts to focus on reproductive differences between man and animals. It is not the authors' intention to provide an extensive review of reproductive health of all animal species with the exception of man. The review ends with a list of suggested further reading for those readers interested in exploring this fascinating topic in greater detail. Instead, this review concentrates on production and companion animals. Production animals must generate their owner a profit, whilst companion animals are often considered to be cherished family members. The owner's attitude to these two types of animal is thus quite different.

Man has interfered with animal reproductive health since domestication started. Man wants animals to breed when 'required' and to stop procreating when the owner dictates. Man also wants to decide the genetic constitution of the offspring. In short, man wants to decide when mating takes place and between which particular animals. These requirements are taken to be a fundamental part of domestication.

This interference in animal reproductive health concentrates on two main issues: breed when needed and stop breeding when not desired. The policy is utilised in two ways in the two main forms of domestication: production animals (e.g. cow, sheep, horse, camel, poultry, fish, dog) and companion animals (mainly dogs and cats, although horse, poultry and fish may be included in this category). The breeding policy is different for the two groups, as is the attitude towards the individual animals concerned. Many techniques used in human reproduction have been developed by veterinarians and vice versa, and the extensive exchange of knowledge has benefited both professions. However, it is rarely possible to extrapolate from one species to another without careful investigation. One must especially beware against taking a drug from the human clinic, adjusting the dose using a formula based on milligrams of drug per kilogram of weight, and expect a valid result when the drug is injected into an animal. Sometimes the effect may be the opposite of that expected in humans (e.g. morphine will make a cat hyperactive).

\section{Reproductive similarities and differences between man and animals \\ Male reproductive system}

The reproductive organs in domesticated male animals are, in general, similar in structure and function to those in man.

J Fam Plann Reprod Health Care 2006; 32(4): 245-248 (Accepted 27 June 2006)

Department of Physiology and Pharmacology, Institute of Medical Biology, University of Southern Denmark, Odense, Denmark

Niels Einer-Jensen, DVM, DScVM, Retired Professor in Physiology

Department of Clinical Studies - Reproduction, The Royal Veterinary and Agriculture University, Copenhagen, Denmark Ronald H F Hunter, PhD, Professor in Reproductive Medicine in Domestic Animals

Correspondence to: Dr Niels Einer-Jensen, Department of Physiology and Pharmacology, Institute of Medical Biology, University of Southern Denmark, Winsløwparken 21, Odense, DK-5000, Denmark. Tel: +45 6619 1949. Fax: +44 4566133479. E-mail: NEiner-jensen@health.sdu.dk
Variations do exist: the penis may be fibro-elastic or tumescent, short or long, thick or thin, and it may have a spiral glans (boar). A penile bone may even be found in some species (e.g. dog). Testes do not descend in some species, making vasectomy (e.g. in elephants) rather difficult. Uni- or bilateral cryptorchidism is not uncommon and is partly genetically based. A boar may ejaculate 200-300 ml of semen more or less directly into the uterus over a 10-minute period, while a bull may ejaculate just a few millilitres during a very brief mating. However, the total number of sperm per ejaculation is more or less the same in the two species. Whilst it is certainly quicker to collect semen for artificial insemination from a bull than from a boar, it may not be easier since a bull is a large, clumsy and potentially dangerous animal. Prolonged (and probably painful) erection can be observed in the dog, making separation from the female difficult (a bucket of cold water is said to facilitate the process). In general, the domesticated male animal will mate and reproduce whenever and wherever possible. Seasonal differences are observed in some species such as sheep and deer in which sperm production may be low or in abeyance outside the breeding season, but demand is high during the mating season. Herd formation is common, and so the males must sometimes fight to gain access to the females. This may be considered a useful form of genetic selection. Organised breeding is a highly regulated event intended to secure optimal genetic combinations. Regulated breeding has successfully produced animals optimised for both production and pleasure; one has only to consider all the specialised breeds of dog that exist today.

\section{Female reproductive system}

The basic components of the female animal reproductive system are the same as in the human: the ovaries, Fallopian tubes, uterus, cervix and vagina. However, a number of key differences are found between humans and female animals, and even between common animal species.

Sexual drive. Female animals do not mate unless they are in oestrus. Oestrus is the rather short period before and during ovulation, dominated by the high oestrogen levels produced by the large follicle(s). After puberty, ovulation takes place every 3 weeks in some domestic animals (e.g. cow, pig), while other species may ovulate only once or a few times every 6 or 12 months. Oestrus should be regarded as 'mating on demand'. Freshly deposited sperm will be available for the newly ovulated egg(s) and should increase the chance of conception. Oestrus is indicated by a change in behaviour, including willingness to mate, and swollen vulvae and fluid discharge. Pheromones will play a role. Male animals will observe the changes and make every effort to copulate with the female animal concerned. Mating can be silent and peaceful, but alternatively may be a noisy and violent affair repeated several times during oestrus, as is the case in cats and lions.

Female dogs (bitches) are noted to bleed in connection with oestrus and ovulation. The high oestrogen level induces endometrial bleeding; it is not a withdrawal bleeding. It is thus not comparable to menstruation since the dog is not a primate.

Ovary. Structures in the ovaries are similar to those of humans. One key difference is the number of ovulations. 
Sheep and cows may have one or a few ovulations, while pigs may ovulate 20 eggs over a period of a few hours. Both ovaries will participate in the ovulatory process. Each site of ovulation will develop into a corpus luteum. Cystic ovaries are not uncommon. There is a major difference between humans and animals as regards regulation of the lifespan of the corpus luteum. In the absence of an embryo, the human corpus luteum will start to degenerate after 10 days. It will need human chorionic gonadotrophin (hCG) to continue progesterone secretion. In contrast, the corpus luteum in some species (e.g. sheep, cow) will persist and produce progesterone for months unless the uterus sends a 'non-pregnant' signal to the ovaries. The non-pregnant hormonal signal is prostaglandin F $2 \alpha$. It is secreted from the endometrium if fertilised eggs (embryos) do not reach the uterus. The luteolytic hormone is transferred locally between the utero-ovarian vein and the ovarian artery. It is thus able to induce luteolysis although the level in peripheral blood is below the limits of detection. Another major difference is the lack of chorionic gonadotrophin in non-primates, although traces of similar substances may be found in some species. Our present knowledge indicates a major difference between recognition of early pregnancy in humans (primates) and non-primates. The authors believe that pregnancies are recognised in both primates (pre-hCG) and non-primates within a day or two following fertilisation, and that a 'common' pregnancy factor will be identified within the next 5 years by means of modern molecular techniques.

Fallopian tubes. In the large farm animals, the Fallopian tubes have a fimbriated infundibulum open to the peritoneal cavity, which tightly embraces the ovary during oestrus. In the horse, the infundibulum forms a bursa. Preovulatory storage of spermatozoa is found in the caudal (distal) portion of the isthmus after mating or artificial insemination. This region is known as the functional sperm reservoir in a wide range of species. Controlled release of viable spermatozoa takes place close to ovulation. The release is under the influence of endocrine signals from the mature Graafian follicle(s).

Uterus. The human uterus has a relatively large corpus and diminutive horns, while most animal species have a short corpus and two long horns. Obviously the long horns make space available for a large number of implantation sites. In most animal species, egg transport through the Fallopian tube will last 3 days and the embryos will be free in the uterus for another 3-8 days according to the species. Strangely, this seems unrelated to the total pregnancy duration (e.g. 21 days of pregnancy in rats and 9 months in cows). A few species (e.g. horse) have a prolonged secretory phase and a relatively late implantation. In some species (e.g. deer) delayed implantation may favour a delayed birth, thus preventing delivery during cold winters. The placenta develops very differently in animal species and the details are outside the scope of this review. In general, the diffusion distance between maternal and fetal blood is greater in animals than in humans. The offspring may therefore lack full immune protection and be dependent on colostrum to survive. Dogs will often develop a vicious, life-threatening pyometritis with retention of content. Antibiotic treatment may not work satisfactorily and oxytocin or prostaglandin treatment may induce peritoneal infections; early surgery may be the only option.

Cervix and vagina. The cervix requires only brief comment. In cattle, the cervix is closely comparable to primate tissues and it is therefore possible to pass an artificial insemination straw or an embryo recovery catheter into the body or horn of the uterus. In contrast, however, this process is rather difficult in sheep. The tumescent glans penis of the stallion will press against the cervix and distend the canal during ejaculation.

\section{Production animals}

Production animals are (as the name indicates) bred and kept to produce offspring, milk and meat, or to carry a load. The owner may like - not to say love - his animals; he may have spent his whole professional life creating a valuable herd of milking cows. Respect for the animals and ethical considerations can be pronounced. The farmer must, however, tread a delicate path; personal feelings should not unduly influence his economic decisions concerning reproductive matters.

Production animals must reproduce effectively in order to maximise profit. Cows and pigs must conceive soon after giving birth. Every day without re-establishing a pregnancy (i.e. an empty day) is a 'wasted' day. The farmer keeps close watch for signs of oestrus and then breeding is attempted. The number of mature male animals is kept at a minimum, generally one per 20-50 females. Alternatively, artificial insemination is routinely carried out using diluted semen from bull or boar stations. The stations utilise the semen effectively and are therefore able to provide highquality, expensive, genetically selected material in a financially competitive manner. The pregnancy rate is usually above $50 \%$ per insemination and is often $>70 \%$. Low fertility may indicate sexually transmitted infections (STIs), malnutrition or sloppy herd management, especially with respect to oestrus control. Low fertility may also be caused by early abortions, either due to endemic infection or malnutrition. A bull or a boar may well be more observant than a farmer in detecting oestrus; vasectomised animals can be used to establish the optimum time for artificial insemination. Keeping a boar in a pen next to the sows may intensify the oestrus symptoms. Animals with repeated oestrus are treated for infertility or sent to the slaughterhouse, as are cows when their milk production fails. Lack of oestrus may be treated with hormone injections to stimulate follicular growth. If a persistent corpus luteum is present it may be removed by manual dislocation during rectal exploration or by injection of a prostaglandin analogue. Such injections are also used to synchronise oestrus in several animals to facilitate artificial insemination or egg transplantation.

No method similar to hCG measurement is currently available for farm animals to confirm the establishment of pregnancy. Pregnancy is detected in cows and sometimes in sows by rectal palpation. Progesterone assays in plasma or milk may be useful but are not in general use. Rectal ultrasound can be helpful but may not be cost effective. Often the farmer will have to await general signs of pregnancy such as an increase in abdominal volume or growth of the udder in young animals. Behavioural changes may be observed; oestrus is a period characterised by irritability and vocal noises, whereas pregnant animals are more relaxed and composed.

High-quality male and female breeding animals are extremely valuable. Intensive research has developed, modified and applied all presently available fertility methods to these animals. Collection of multiple eggs from hyperstimulated ovaries, splitting of four- to eight-cell stages into several embryos, freezing of eggs or embryos, and cloning are available. Methods applied in the male animal seem to be cheaper and easier to use. Artificial insemination is more or less standard in the dairy industry 
and in pig production. Both male and female methods assure a rapid distribution of productive gene combinations to large numbers of ordinary animals.

Parturition is an exciting event; newborns from large animals may weigh $50 \mathrm{~kg}$ and require hard physical work from both the mother and the farmer. Long legs (e.g. sheep, cow, horse) may require manual adjustment. Labour in horses is associated with violent contractions and any problem may demand rapid assistance from a veterinarian to prevent ruptures. Caesarean sections are common; these may be performed in a 'standing and eating' cow under local and (low-dose) spinal anaesthesia. Improved surgical techniques have diminished the need for embryotomy and it is rarely performed these days. Twins are a mixed blessing in cattle since a female fetus is exposed to male hormones if the other fetus is a male with the result that the female will acquire 'nymphomaniac' behaviour and will not reproduce later in life. Such an animal is termed a 'Free Martin' and is distinct from an intersex animal possessing ovotestes. Colostrum is essential to protect the newborn against infections and to stimulate gastric activity. Lactation is energy demanding: a cow may produce 40-80 litres of milk per day and a sow must feed up to 20 hungry piglets. The farmer will provide high-energy fodder to cover the milk production but the animal may still lose weight nonetheless. Intravenous injection of oxytocin will help the mother to deliver milk to the offspring.

Castration of male animals is common. As a consequence, male aggressive behaviour is decreased, and the sedate animal will grow better and require less food and be easier to use for riding or pulling loads. Pork meat from entire males may have an unpleasant taste and odour, which is not present in meat from castrated or female animals. Chemical castration with diethylstilbestrol is no longer permitted.

Specific pathogen-free (SPF) animals live behind a 'bacteria-tight' barrier. They grow faster, demanding less food per kilogram increase in body weight. Removing piglets by Caesarean section and placing them in a sterile incubator is the method of production. The piglets are exposed to defined bacterial cultures and are later used to produce the SPF production animals.

\section{Companion animals}

Companion animals (primarily dogs and cats) are kept for the owner's pleasure and certainly not for profit. The attitude towards such animals is therefore quite different to that of production animals. Companion animals are often treated as members of the family. The cost of maintaining a healthy animal can be high; we are often amazed by the amount of money people are willing to invest in their pets. In cats the urge to reproduce is strong. A female cat in oestrus will break the daily household rules and disappear from the family home, and the male cats in the neighbourhood will smell her pheromones miles away and take up the pursuit. Family planning is thus an important aspect of keeping domestic cats.

Companion animals are supposed to 'behave'. Both castrated males and females are gentler in nature and easier to handle than are their gonadal hormone-driven counterparts. The tendency to disappear temporarily from the family home may decrease. Both cats and dogs are territorial animals; castrated animals will pick fewer fights. They may also smell less. Owners may not want their animal to breed nor may they want the extra effort involved in raising newborn offspring. Castration of male and female companion animals is common, as is castration of young stallions. One may observe a conflict between the animal's normal behaviour and the owner's expectations. There is, however, an additional ethical conflict, namely how much surgery is acceptable: removing the tail, diminishing the ears, removing the claws (in cats), inserting silicone testes to make the animal look normal, and other such modifications. The timing of castration is crucial; owners generally prefer to have an animal with a well-developed body and mature behaviour. Thus the animal should be beyond the kitten stage but not yet at the stage of being a roaring, fighting male, marking its territory with urine. Any acquired (bad) behaviour tends to remain despite castration.

Vasectomy and salpingectomy are uncommon in companion animals; usually the gonads are also removed. This is partly a tradition but is also a necessity if 'bad' behaviour is part of the animal's problem. Sterilisation may be used to diminish population growth in wild or semi-wild populations (e.g. feral cats or in national parks). In dogs, the uterus is normally also removed during the procedure to protect the animal against pyometritis.

Cryptorchidism is not uncommon in animals such as dogs and horses; one should always count the testes before buying or selling an animal. The retained organ may have a high testosterone production, resulting in unpleasant - not to say intolerable - male behaviour. As in the human species, the retained testis may develop cancer and should be removed whether it is retained in the inguinal canal or abdomen. Since the defect may be inherited, the male should not be used for breeding.

Infertility and low fertility are quite common in companion animals. In general, one should not be too eager to breed such animals since low fertility may be an inherited trait. In rare cats and dogs it may be a sign of inbreeding and may be associated with other adverse genetic traits. Male animals with low fertility are rarely treated; however, all methods from the human fertility clinic can in principle be applied, whether the reason is low sperm quality, infection or lack of sexual drive. In females, the obvious sign of infertility is the absence of oestrus. Oestrus can be induced with an oestradiol benzoate injection, but such cycles are infertile since the ovaries are not stimulated. Follicle growth and ovulation may be induced in cats and dogs by means of injections of folliclestimulating hormone/luteinsing hormone preparations using the same principle (but not dose) as is used in humans. Sexovid ${ }^{\circledR}$ (with clomiphene-like effects) has been used successfully to induce fertile cycles in dogs by one of the authors but the substance is no longer available. Infertility, despite a normal oestrus, may be due to treatable genital infections or structural malformations.

It is difficult to detect an early pregnancy; in general one simply has to wait and see. Pseudo-pregnancies are common in dogs; behavioural changes including nest building and mammary growth may be seen, with the the dog putting up a very convincing show. No hCG-type assay is available; however, progesterone analyses and ultrasound are useful. Careful palpation is helpful if the animal is lean and co-operative; use 'soft' fingers to avoid abortion. Behavioural changes and milk in the glands will indicate that parturition is approaching, whereupon the delivery should be supervised. Delivery may take hours since many puppies or kittens may need to be born; however, delivery of individual offspring is normally very fast. Most animals will deliver by themselves; only rarely is support with a pair of forceps or two fingers needed. A dead fetus interspaced between a series of live newborns is common. It is important to palpate the uterus or use X-rays or ultrasound to ascertain that delivery is complete, since the total number of fetuses in the uterus is not normally 


\section{REVIEW}

known in advance. Serious delays or other problems during delivery will indicate that a Caesarean section is needed. Many mothers want to eat the placentae; this should be permitted. Only a small proportion of animals will fail to recognise their offspring or feed them; when it does occur the problem is mostly lack of experience. Even experienced mothers will sometimes abandon weak newborns; it is a cruel process to watch. The owner may be able to reduce the problem by keeping the offspring warm, massaging the mother's breast and comforting her.

Family planning methods in companion animals are, in principle, similar to those used by humans. They are, however, used with many modifications. Several STIs exist in both companion and farm animals; even a variant of AIDS is found in cats. Breeding animals should be checked for STIs. Condoms are not used for obvious reasons. Intrauterine devices (IUDs) are not used in animals, although the oldest example of an IUD is stone pebbles inserted into the uterus in camels on long caravan journeys. Hormonal contraception, especially long-acting gestagens, is common in dogs and cats. Oestrogens should be avoided. A weekly Perlutex ${ }^{\circledR}$ tablet (1-medroxyprogesterone acetate, $5 \mathrm{mg}$ ) will prevent oestrus and pregnancy in a cat. Oestrus can be stopped by using higher doses. A subcutaneous injection of Perlutex every 6 months will prevent oestrus and pregnancy in dogs. If the injection is given for the first time close to pro-oestrus or oestrus it may induce pyometritis. It is, however, generally safe to interrupt oestrus in dogs with a daily Perlutex tablet and then continue with 6-monthly injections. If a dog has an unwanted mating it may be treated with a subcutaneous injection of oestradiol benzoate within the first few days. The dog should be observed carefully during subsequent weeks on account of a risk of pyometritis. This procedure should be used with caution, however, since pyometritis poses a serious health risk for dogs.

Well-planned cat and dog breeding is a rewarding job for those very committed people who do not expect to become rich from this time-consuming activity. From time to time they will experience problems with breeding and parturition, and general problems with all types of infection. It is important to select healthy, fertile and attractive animals possessing a good temperament. It is also important to ensure that the breeding animals have access to a good diet and are maintained in rigorously hygienic living conditions. This combination is often difficult to find, but any potential purchaser of a puppy should be critical of the kennel and the kennel owner. Potential purchasers should also be clear about the preferred sex, temperament, and so on, of their prospective family member and only select a puppy that matches their requirements.

\section{Exotic animals}

Man keeps all sorts of unusual animals as companion animals. Environmental degradation currently threatens many wild animal species. It is possible but expensive to provide reproductive health facilities and the means of survival for many of these special cases, however a huge bank of knowledge is required. Zoos and national parks may have special breeding programmes such as artificial insemination and in vitro fertilisation using fresh or frozenthawed sperm and eggs. Exchange of animals between different facilities and locations is one way in which to broaden the genetic pool and avoid inbreeding in small populations of animals.

Statements on funding and competing interests

Funding. None identified.

Competing interests. None identified.

\section{Suggested further reading}

There are numerous textbooks and reference books available on production and domestic animals. The titles listed below represent the authors' personal recommendations of books suitable for interested readers who want to explore this fascinating subject in greater detail.

1 Pineda MH, Dooley MP(eds). McDonald's Veterinary Endocrinology and Reproduction [textbook]. Oxford, UK: Blackwell Publishing, 2002.

2 Wright JG, Arthur GH, Noakes DE, Pearson H, Parkinson TJ (eds). Veterinary Reproduction and Obstetrics [standard textbook for veterinary students]. Oxford, UK: W B Saunders, 1995.

3 Senger PL (ed.). Pathways to Pregnancy and Parturition [rather basic textbook]. Pullman, WA: Current Conceptions Inc., 1997.

4 Hafez ESE, Hafez B (eds). Reproduction in Farm Animals [standard textbook for veterinary students]. Oxford, UK: Blackwell Publishing, 2000.

5 Cupps P (ed.). Reproduction in Domestic Animals. London, UK: Academic Press, 1990

6 Feldman EC, Nelson RW. Canine and Feline Endocrinology and Reproduction (3rd edn). Oxford, UK: W B Saunders, 2003

7 Davies Morel MCG (ed.). Equine Reproductive Physiology, Breeding and Stud Management (2nd edn). Wallingford, UK: CABI Publishing, 2002

8 Cole DJA, Foxcroft GR (eds). Control of Pig Reproduction. London, UK: Butterworths, 1988

9 Adams HR (ed.). Veterinary Pharmacology and Therapeutics [basic and applied principles of treatment]. Ames, IA: Iowa State University Press, 2001.

10 Line S, Kahn CM (eds). The Merck Veterinary Manual (9th edn). Oxford, UK: Elsevier (Merck Publications), 2005.

In addition, Reproduction and Journal of Reproduction and Fertility have published a number of supplements comprising research papers on reproduction in animals. (NB. Bold numbers are the supplement number and Roman numerals indicate that older supplements from other conferences are available.)

Reproduction: Reproduction in domestic ruminants V, 61 (2003); Fertility control in wildlife, 60 (2002); Large animals as neuroendocrine models, 59 (2000); Control of pig reproduction VI, 58 (2001).

Journal of Reproduction and Fertility: Advances in reproduction in dogs, cats, and exotic carnivores, 57 (2001); Equine reproduction VII, 56 (2000); Reproduction in domestic ruminants IV, 54 (1999); Control of pig reproduction V, 52 (1997).

Editor's Note

A forthcoming review article will focus on reproductive health in zoo/wild animals.

\section{LETTERS TO THE EDITOR}

Letters to the Editor are welcome and generally should not exceed 600 words or cite more than five references. For comments on material published in the most recent issue of the Journal, correspondence should be received within 4 weeks of dispatch of that Journal to be in time for inclusion in the next issue. When submitting letters correspondents should include their job title, a maximum of two qualifications and their address(es). A statement on competing interests should also be submitted for all letters. Letters may be submitted to the Editor or the Journal Editorial Office (details on page 209). 